\title{
Editorial
}

\section{The future role of the Scientific Committee on Antarctic Research}

$\mathrm{T}$ he Scientific Committee on Antarctic Research (SCAR) is now more than 60 years old and about to embark on the development of a new Strategic Plan. The immediate question, therefore, is what path should SCAR follow? SCAR has long had two major roles. The first is science coordination. SCAR initiates and facilitates research in, from and about Antarctica and the Southern Ocean, particularly research concerned with the role of the region in the changing Earth System. The second is the provision of evidence-based scientific advice to decision-makers in the Antarctic Treaty System (ATS) and to those in other bodies such as those of the United Nations. Here, SCAR is in a unique position.

SCAR is the only observer to the Antarctic Treaty Consultative Meetings specifically mentioned in the Protocol on Environmental Protection to the Antarctic Treaty (Article 10(2)) as a provider of independent advice to the Antarctic Treaty Consultative Parties (ATCPs). In this role, SCAR is widely appreciated. At their 42nd meeting, through Resolution 7 (2019) the ATCPs acknowledged '... with gratitude SCAR's enduring and crucial role in providing objective and independent scientific advice to support and inform the work of the ATCM [Antarctic Treaty Consultative Meeting] and the CEP [Committee on Environmental Protection] ...'.

SCAR is also an affiliated body of the International Science Council and makes substantive contributions elsewhere such as by facilitating inputs and contributing to the reports of the Intergovernmental Panel on Climate Change (IPCC) and the Intergovernmental Science-Policy Platform on Biodiversity and Ecosystem Services (IPBES).

SCAR's advice can thus readily be provided to the ATS and to other bodies in ways not constrained by the jurisdictions of international agreements. In other words, the $60^{\circ} \mathrm{S}$ limit of the Antarctic Treaty and the Antarctic Convergence limit of the Convention on the Conservation of Antarctic Marine Living Resources place no restrictions on SCAR's science or the advice it provides. The Earth System does not recognize political boundaries, and nor should research and scientific advice about it. Not only do SCAR's founding documents make clear that its research includes the sub-Antarctic, but SCAR-facilitated research has long recognized the significance of global teleconnections. Antarctica and the Southern Ocean are integral parts of the Earth System.

In the context of SCAR's forthcoming Strategic Plan, these two major roles - science facilitation and advice should, of course, remain foundational. Yet SCAR's three new Scientific Research Programmes (SRPs) should take centre stage because of their international significance. These new SRPs focus on 1) ice-sheet instabilities, thresholds and sea-level rise (INSTANT), 2) short-term (1-30 years) variability in climate (AntClim ${ }^{\text {now }}$ ) and 3) patterns and processes in and the conservation of marine and terrestrial biodiversity given changes in the region (Ant-ICON).

These SRPs will deliver scientific outcomes that are arguably amongst the most crucial for the future of modern society, that will substantially improve our understanding of climate variability in the region, and that will inform decisions to ensure a favourable future outlook for the extraordinary biodiversity that is a hallmark of the Antarctic and Southern Ocean. Moreover, the SRPs have been purposefully designed such that their outcomes and findings will enable SCAR to continue to grow its highly regarded evidence-based advice to the ATS and to others. The potential societal benefits are considerable at a time when appreciation for the value and benefit of policy action informed by scientific evidence has deepened significantly.

That advice must necessarily have a global, Earth System context. Only through globally integrated research and policy decisions that recognize the unity of the Earth System are the future envisaged by the Sustainable Development Goals and one that avoids dangerous climate change likely to be realized. Yet SCAR's research and advice must by necessity also consider the regional context - one that includes a region set aside 'as a natural reserve, devoted to peace and science' and the significant aspirations for globally leading conservation that this implies. In addressing these requirements, SCAR's advice must be characterized by clarity of presentation founded on the best available evidence. At times, this may result in some unintended political discomfort. Yet that discomfort will remain far less than what will be coming if the considerable challenges to the Earth System, Southern Ocean and Antarctica, largely as a consequence of climate change, are not avoided because the implications of the scientific evidence have not been made clear.

Therefore, integration of the three new SRPs with the provision of timely, clear and reliable advice to the ATS and a range of other bodies must serve as a core to SCAR's new Strategic Plan. Obviously, SCAR facilitates a diverse 
portfolio of research through the dedicated work of its members and volunteers - and it should continue to do so. But only through focused, foundational support of its new SRPs and continual growth of its science advice role will SCAR provide the research and policy service most needed now by society. Our lives, and those of the species with which we share the planet, depend on it.

STEVen L. ChOWN Securing Antarctica's Environmental Future, Monash University, Victoria 3800, Australia 